\title{
Normal serum alanine aminotransferase and non-alcoholic fatty liver disease among Korean adolescents: a cross-sectional study using data from KNHANES 2010-2015
}

Yunkoo Kang, Sowon Park, Seung Kim and Hong Koh ${ }^{*}$

\begin{abstract}
Background: Non-alcoholic fatty liver disease (NAFLD) is complicated disease and increasing worldwide. Previously, many studies of NALFD prevalences have used alanine aminotransferase (ALT) of $>40 \mathrm{U} / L$ to define NAFLD, although that is too high to be reliable among adolescents. This study aimed to define the upper normal limit of ALT among Korean adolescents, and use it to estimate the prevalence of NAFLD, based on data from the Korea National Health and Nutrition Examination Survey (KNHANES).

Methods: Data were obtained from 1785 healthy adolescents (916 boys and 869 girls, 10-18 years old) who participated in the KNHANES during 2010-2015. The International Diabetes Federation metabolic syndrome criteria for adolescents were used to exclude participants with metabolic syndrome components. Furthermore, participants who previously had diseases related to low HDL levels, high TG levels, diabetes, or very low/high body mass index and hepatitis B were excluded. The 95th percentiles level of ALT from healthy participants were evaluated. The definition of NAFLD was overweight status ( $\geq 85$ th percentile of body mass index) plus elevated ALT levels (95th percentile).

Results: The upper normal ALT were 24.1 U/L for boys and 17.7 U/L for girls. Based on these values, the estimated prevalences of NAFLD in 2015 were $8.9 \%$ among adolescents.

Conclusion: Defining the upper normal limit of ALT can be adjusted for each sex and ethnics in the general population. ALT laboratory thresholds used for children should be re-examined. The physicians should be aware not to underdiagnose NAFLD patient even ALT level is $<40 \mathrm{U} / \mathrm{L}$.
\end{abstract}

Keywords: Non-alcoholic fatty liver disease, Alanine aminotransferase, Korea, Upper normal limit

\section{Background}

\section{General and specific background}

The prevalence of non-alcoholic fatty liver disease (NAFLD) is increasing worldwide. [1] Therefore, it is necessary for obese adolescents to be actively examined for NAFLD. And symptoms and clinical signs, laboratory and radiological imaging test, and liver biopsy is needed to make diagnosis finally as NAFLD. [2, 3] But in general population, it is not possible to perform all diagnostic procedures for each

\footnotetext{
* Correspondence: khong@yuhs.ac

Department of Pediatrics, Severance Children's Hospital, Severance Pediatric Liver Disease Research Group, Yonsei University College of Medicine, 50-1 Yonsei-ro, Seodaemun-gu, Seoul 03722, Republic of Korea
}

people. Therefore, Alanine aminotransferase (ALT) is used to find prevalence in general populations. ALT is an enzyme that is found in the cytosol of hepatocytes, and blood levels of ALT increase after liver injury [4]. Thus, blood testing for ALT is used globally as a minimally invasive and inexpensive tool for detecting chronic liver diseases, such as non-alcoholic fatty liver disease (NAFLD) [1].

\section{Debating issue}

However, the reference ranges for normal ALT vary widely across different laboratories and populations [1]. Among adults, the upper normal limit of ALT was derived from healthy Italian blood donors, with values of

(c) The Author(s). 2018 Open Access This article is distributed under the terms of the Creative Commons Attribution 4.0 International License (http://creativecommons.org/licenses/by/4.0/), which permits unrestricted use, distribution, and reproduction in any medium, provided you give appropriate credit to the original author(s) and the source, provide a link to the Creative Commons license, and indicate if changes were made. The Creative Commons Public Domain Dedication waiver (http://creativecommons.org/publicdomain/zero/1.0/) applies to the data made available in this article, unless otherwise stated. 
$30 \mathrm{U} / \mathrm{L}$ for men and $19 \mathrm{U} / \mathrm{L}$ for women [5]. Among American adolescents, the upper normal limit of ALT was estimated by the National Health and Nutrition Examination Survey to be $25.8 \mathrm{U} / \mathrm{L}$ for boys and $22.1 \mathrm{U} /$ L for girls [6, 7].

\section{Specific purpose of this study}

Nevertheless, population differences indicate that these values may not be the same among Korean adolescents. However, the method used to define NAFLD in this study is only tools to estimated NAFLD in public populations, so this result cannot directly be used in clinic. But result of this study will give clues to evaluate and manage NAFLD in clinic. Therefore, the present study aimed to estimate the upper normal limits of ALT among Korean adolescents, as well as the prevalences of NAFLD based on those values.

\section{Methods}

\section{Database}

The present study evaluated data from the 2010-2015 Korea National Health and Nutrition Examination Survey (KNHANES). These annual cross-sectional surveys are performed using multi-stage probability samples that are representative of the general Korean population. The data of KNHANES surveys are available at http://knhanes.cdc.go.kr/.

\section{Study sample.}

During 2010-2015, 48,482 individuals participated in the KNHANES. The present study included participants who were 10-18 years old, but excluded participants with missing data and metabolic syndrome components, based on the International Diabetes Federation consensus definition (www.idf.org). Furthermore, 10-15-year-old participants were excluded if they had a waist circumference of $\geq 90$ th percentile for sex and age, triglyceride (TG) levels of $\geq 150 \mathrm{mg} / \mathrm{dL}$, high density lipoprotein (HDL) levels of $<40 \mathrm{mg} / \mathrm{dL}$, systolic blood pressure (SBP) of $\geq 130 \mathrm{mmHg}$, diastolic blood pressure (DBP) of $\geq 85 \mathrm{mmHg}$, and glucose levels of $\geq 100 \mathrm{mg} / \mathrm{dL}$. Moreover, participants who were 16-18-years-old were excluded if they had a waist circumference of $\geq 90 \mathrm{~cm}$ or HDL levels of $<40 \mathrm{mg} / \mathrm{dL}$ (boys), a waist circumference of $\geq 80 \mathrm{~cm}$ or HDL levels of $<50 \mathrm{mg} / \mathrm{dL}$ (girls), TG levels of $\geq 150 \mathrm{mg} / \mathrm{dL}$, SBP of $\geq 130 \mathrm{mmHg}$, DBP of $\geq 85 \mathrm{mmHg}$, and glucose levels of $\geq 100 \mathrm{mg} / \mathrm{dL}$. Finally, we excluded participants who previously had diseases related to low HDL levels, high TG levels, diabetes, or very low/high body mass index (BMI, <5th percentile or $>85$ th percentile) [8]. Furthermore, participants who had hepatitis B infection were excluded. Thus, 1785 healthy participants (916 boys and 869 girls) were included in the analysis to determine the upper normal limit of ALT. For the present study, NAFLD was defined as being overweight ( $\geq 85$ th percentile of BMI) plus having elevated ALT ( 295 th percentile, $24.1 \mathrm{U} / \mathrm{L}$ for boys and $17.7 \mathrm{U} / \mathrm{L}$ for girls). A total of 4149 participants (2226 boys and 1923 girls) were included in the analyses to estimate the prevalence of NAFLD (Figure 1) [9].

\section{Statistical analysis}

All statistical analyses were performed using SPSS software (version 23.0; IBM Inc., Armonk, NY). Categorical

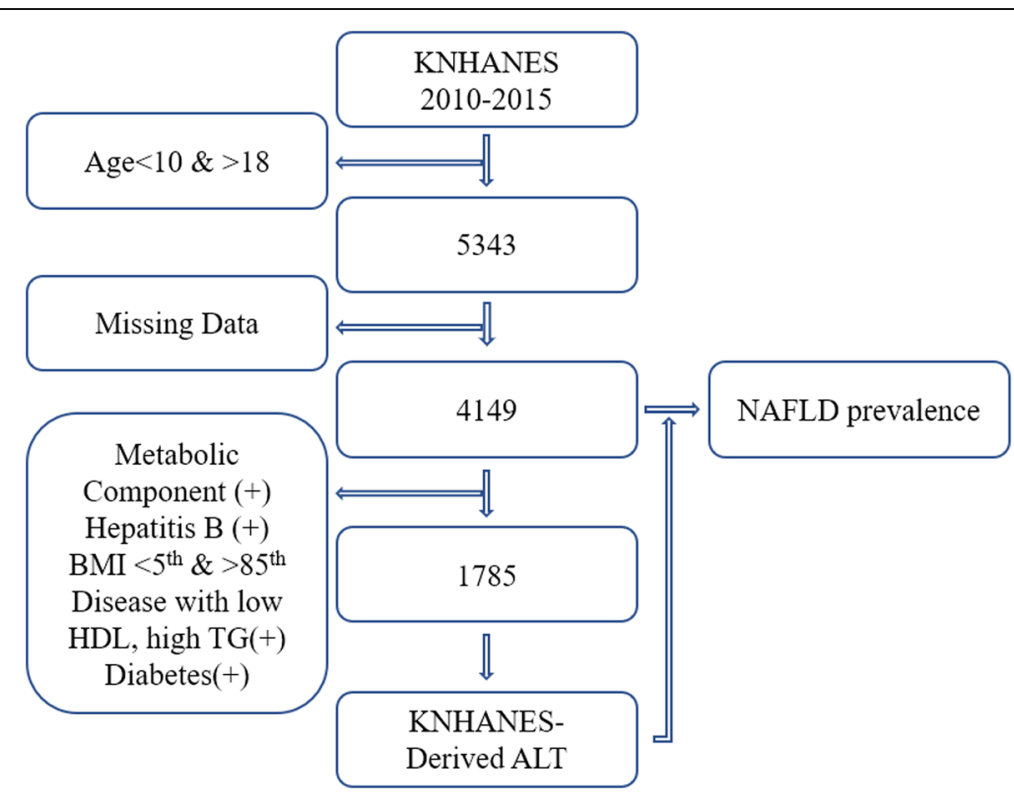

Fig. 1 Flow chart for participant selection (916 boys, 869 girls) 
data were collected for healthy participants (without metabolic syndrome components), and continuous laboratory and anthropometry data were expressed as mean \pm standard error. The 95th percentiles for ALT were estimated for each sex using weighted analysis, and those values were used to estimate the prevalences of NAFLD among adolescents who participated in KNHANES and had available BMI and ALT data.

\section{Results}

\section{Characteristics of the healthy participants}

The characteristics of the 1785 healthy participants (916 boys and 869 girls) are shown in Table 1. All characteristics appeared to be within the normal ranges, and the mean ALT levels were 14.2 U/L for boys and 10.9 U/L for girls. The 95th percentiles for ALT among healthy participants were $24.1 \mathrm{U} / \mathrm{L}$ for boys and 17.7 U/L for girls (Figure 2a).

\section{Prevalence of elevated ALT among Korean adolescents} A total of 4149 KNHANES participants had available ALT and BMI data. Among all participants during 20102015, the estimated prevalence of elevated ALT levels was $10.9 \%$ (95\% confidence interval [CI]: 9.8-12.1\%). During 2015, the overall estimated prevalence of elevated ALT levels was 13.7\% (95\% CI: 11.0-15.5\%). In addition, the estimated prevalences of elevated ALT levels during 2015 were $14.9 \%$ (95\% CI: $11.0-19.8 \%$ ) for boys and $12.3 \%$ (95\% CI: 8.6-17.3\%) for girls (Figure $2 b$ ).

\section{Prevalence of NAFLD among Korean adolescents}

The prevalence of NAFLD was estimated using the 95th percentile values for ALT (24.1 U/L for boys and $17.7 \mathrm{U} /$

Table 1 The characteristics of healthy KNHANES participants during 2010-2015

\begin{tabular}{llll}
\hline & Total & Male & Female \\
\hline Age $($ year $)$ & $13.98 \pm 0.07$ & $13.98 \pm 0.10$ & $13.99 \pm 0.10$ \\
SBP $(\mathrm{mmHg})$ & $105.39 \pm 0.28$ & $107.01 \pm 0.41$ & $103.78 \pm 0.35$ \\
DBP(mmHg) & $64.99 \pm 0.27$ & $64.98 \pm 0.41$ & $65.00 \pm 0.32$ \\
Height(cm) & $160.12 \pm 0.30$ & $163.54 \pm 0.50$ & $156.70 \pm 0.31$ \\
Weight(kg) & $50.35 \pm 0.29$ & $53.06 \pm 0.49$ & $47.63 \pm 0.30$ \\
Waist(cm) & $66.24 \pm 0.18$ & $67.77 \pm 0.27$ & $64.70 \pm 0.22$ \\
BMl(kg/m $\left.{ }^{2}\right)$ & $19.41 \pm 0.06$ & $19.56 \pm 0.10$ & $19.26 \pm 0.07$ \\
Glucose(mg/dl) & $88.52 \pm 0.17$ & $89.00 \pm 0.22$ & $88.04 \pm 0.22$ \\
Cholesterol(mg/dl) & $158.80 \pm 0.74$ & $152.82 \pm 1.01$ & $164.79 \pm 1.06$ \\
HDL(mg/dl) & $55.06 \pm 0.29$ & $53.54 \pm 0.39$ & $56.57 \pm 0.40$ \\
TG(mg/dl) & $68.76 \pm 0.78$ & $66.42 \pm 1.09$ & $71.11 \pm 1.07$ \\
AST(U/L) & $18.60 \pm 0.15$ & $20.02 \pm 0.22$ & $17.18 \pm 0.17$ \\
ALT(U/L) & $12.56 \pm 0.18$ & $14.18 \pm 0.30$ & $10.94 \pm 0.19$ \\
\hline
\end{tabular}

Data are presented as mean \pm standard error

SBP Systolic blood pressure, DBP Diastolic blood pressure, BMI Body mass index, HDL High-density lipoprotein, TG Triglycerides, AST Aspartate aminotransferase, ALT Alanine aminotransferase
L for girls) plus the age- and sex-specific 85th percentile values for BMI. During 2015, the overall prevalence of NAFLD was $8.9 \%$ (95\% CI: 6.7-11.6\%), with prevalences of $10.8 \%$ (95\% CI: $7.7-15.0 \%$ ) among boys and 6.6\% (95\% CI: 4.0-10.9\%) among girls (Figure 3).

\section{Discussion}

The present study evaluated data from the general population of healthy Korean adolescents to estimate the upper normal limit of ALT. The results indicate that the upper limits (95th percentiles) for estimating the prevalence of NAFLD in this population were $24.1 \mathrm{U} / \mathrm{L}$ for boys and 17.7 U/L for girls. These values are similar to, albeit lower than, the values among American adolescents (25.8 U/L for boys, $22.1 \mathrm{U} / \mathrm{L}$ for girls) [6].

The present study used the upper normal limit of ALT to estimate the prevalence of NAFLD, which revealed values of $10.8 \%$ among boys and $6.6 \%$ among girls in 2015. In contrast, use of the previous standard values (30 U/L for boys and $19 \mathrm{U} / \mathrm{L}$ for girls) generated estimated NAFLD prevalences of $6.7 \%$ among Korean boys and 5.1\% among Korean girls in 2015 [10] [5, 11]. Thus, the prevalence of NAFLD in this population appears to be unexpectedly high, and we recommend aggressive management for patients who may have undetected NAFLD based on the previous standard ALT values.

The prevalence of NAFLD (elevated ALT levels (> $30 \mathrm{U} / \mathrm{L})$ ) was $3.6 \%$ in boys and $2.8 \%$ in girls from 1594 adolescents aged 10 to 19 years using the 1998 KNHANES. [12] The prevalence of NAFLD in Chinese children found out to be $9.03 \%$ with ALT thresholds > $25.8 \mathrm{U} / \mathrm{L}$ for boys $>22.1 \mathrm{U} / \mathrm{L}$ for girls using China Health and Nutrition Surveys. [13] Although there is a lack of uniformity in the data, but similar results were obtained with studies conducted in China from our study. Emma et al. pooled prevalence of NAFLD as 7.6\% (95\%CI: 5.5 to $10.3 \%)$ using meta-analysis and showed that it did not differ by geographical region among children and adolescents. [14, 15] Even with heterogenicity of defining NALFD across studies, it seems that the prevalence of NAFLD increase with time and has globally similar prevalence.

This study had some important limitations. First, we used elevated ALT plus elevated BMI as criteria to diagnose NAFLD. Some NAFLD patients may have normal ALT levels and it does not parallelly match the histological severity of NAFLD in children. [16] So, in order to diagnose NALFD, imaging investigations or histology confirmation should be included. However, we have not been able to confirm NAFLD using imaging investigation in this cohort study as it has not been used in every cycle of KNHANES. Therefore, it could not be included in the criteria of NAFLD in this study. However, ALT 

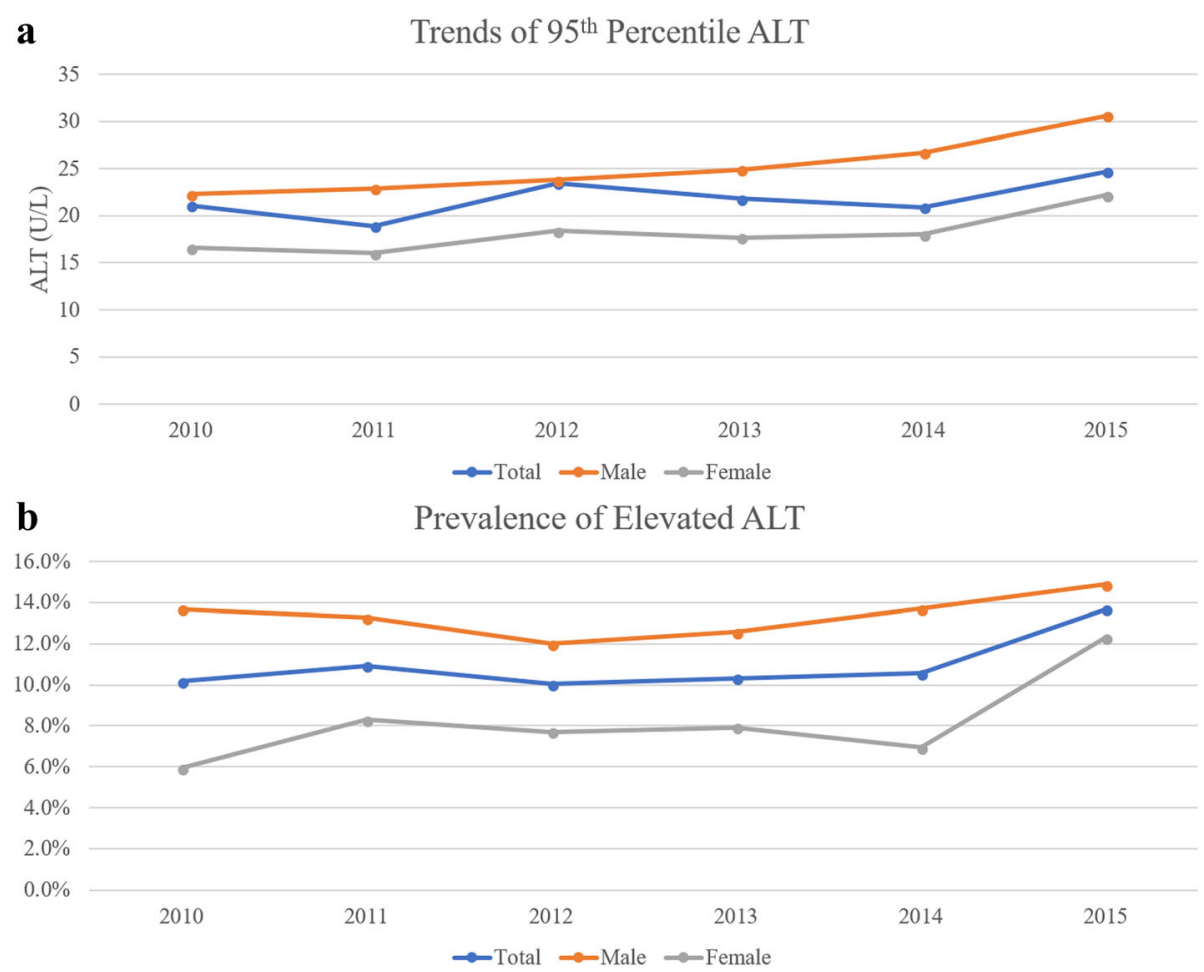

Fig. 2 Trends in the 95th percentiles for alanine aminotransferase (a) and the prevalence of participants with elevated alanine aminotransferase (>95th percentile) (b)

has been recommended as a screening tool for NAFLD and has previously been used for population-based epidemiological studies. [7, 8, 17] Furthermore, the upper normal limits of ALT (24.1 U/L for boys and 17.7 U/L for girls) should be validated using liver biopsies $[7,18]$. Second, the KNHANES data do not include information regarding the use of hepatotoxic medication. Third, although ours is the most recent study to estimate the sex-specific upper normal limits of ALT among Korean adolescents, additional criteria should be considered when using ALT levels to estimate the prevalences of other chronic liver diseases in Korea. As we mentioned at the beginning, NAFLD should be diagnosed using liver biopsy or imaging studies. So, results from present study cannot directly be used in clinic level.

However, even with these limitation, this is first study to define upper normal ALT level of adolescents by using KHANES data. And according to our data, prevalences of adolescents NAFLD in Korea might be more serious than we used to think. As the result of this study shows normal value of ALT can varies by some circumstances, ALT laboratory thresholds used for children should be re-examined. The physicians should be aware not to underdiagnose NAFLD patient when ALT level is

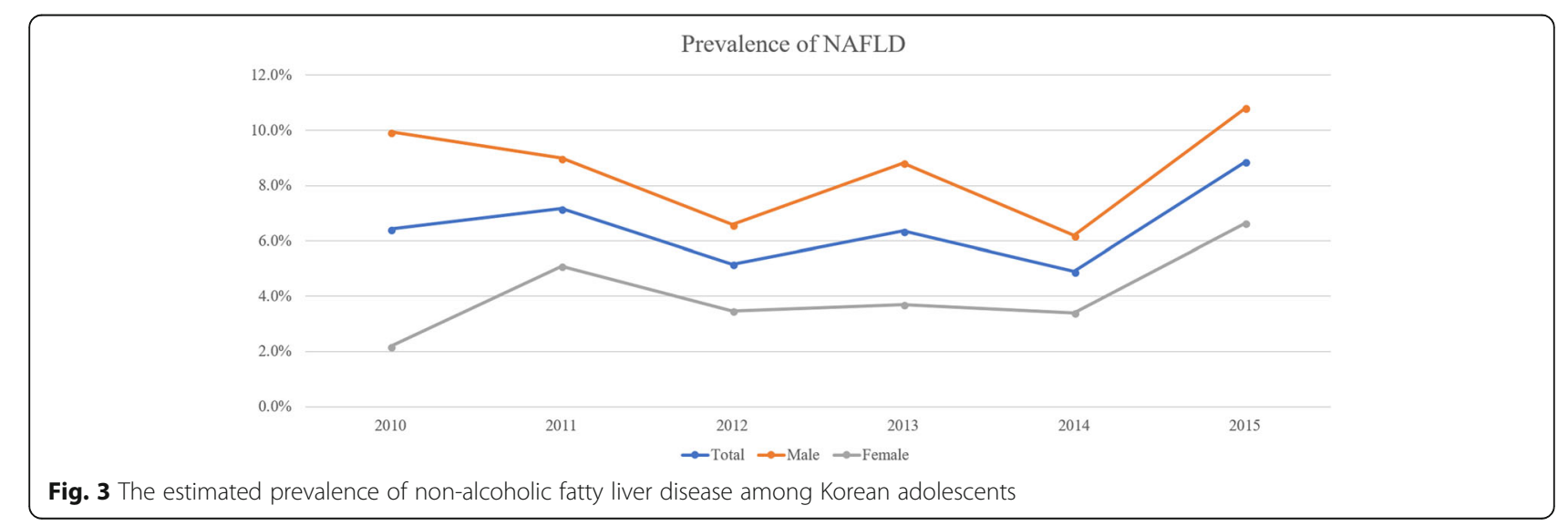


in normal value we used to use in clinic. However, the defining NAFLD (BMI $\geq 85$ th percentile plus elevated ALT) used in the study is only tools to estimate NAFLD in public populations, so this upper normal ALT results cannot directly be used in clinic. We hope these results can give clues that ALT level can be adjusted for each sex and ethnics. And ALT could be more useful tool to determine who may need more detailed medical examinations for NAFLD if ALT is adjusted specific for each ethnics and sex.

\section{Conclusions}

The upper normal level of ALT in Korea were 24.1 U/L for boys and 17.7 U/L for girls in our study. And based on the weighting of the KNHANES design, our estimates indicate that NAFLD may be present in year 2015, approximately 282,981 adolescent boys and 159,154 adolescent girls. Thus, additional care is needed to identify Korean adolescents with undetected NAFLD and its complications. And further study is needed for evaluating sensitivity and specificity of upper normal level of ALT from this study.

\section{Abbreviations}

BMI: Body mass index; DBP: Diastolic blood pressure; HDL: High density lipoprotein; KNHANES: Korea National Health and Nutrition Examination Survey; NAFLD: Non-alcoholic fatty liver disease; SBP: Systolic blood pressure; TG: Triglyceride

\section{Availability of data and materials}

The all raw data of survey is available http://knhanes.cdc.go.kr/. The datasets during and/or analyzed during the current study available from the corresponding author on reasonable request.

\section{Authors' contributions}

YK and HK conceived and led the design of the study, analyses, and drafting of the article. YK wrote the first draft of the paper. YK, SP, SK, and HK contributed to the discussion of the results, revisions, and approval of the manuscript. YK conducted the extraction of data and data analysis. All authors read and approved the final manuscript.

\section{Ethics approval and consent to participate}

The KNHANES surveys (http://knhanes.cdc.go.kr/) are approved by the Korean Center for Disease Control (2010-02CON-21-C, 2011-02CON-06-C, 2012-01EXP-01-2C, 2013-07CON-03-4C, 2013-122EXP-03-5C, and 2015-01-02$6 C)$, and all participants provide written informed consent.

\section{Consent for publication}

Not applicable.

\section{Competing interests}

The authors declare that they have no competing interests.
2. Oh H, Jun DW, Saeed WK, Nguyen MH. Non-alcoholic fatty liver diseases: update on the challenge of diagnosis and treatment. Clin Mol Hepatol. 2016;22(3):327-35

3. Janczyk W, Socha P. Non-alcoholic fatty liver disease in children. Clin Res Hepatol Gastroenterol. 2012;36(3):297-300.

4. Kim WR, Flamm SL, Di Bisceglie AM, Bodenheimer HC. Public policy Committee of the American Association for the study of liver D: serum activity of alanine aminotransferase (ALT) as an indicator of health and disease. Hepatology. 2008;47(4):1363-70.

5. Prati D, Taioli E, Zanella A, Della Torre E, Butelli S, Del Vecchio E, Vianello L, Zanuso F, Mozzi F, Milani S, et al. Updated definitions of healthy ranges for serum alanine aminotransferase levels. Ann Intern Med. 2002;137(1):1-10.

6. Schwimmer JB, Dunn W, Norman GJ, Pardee PE, Middleton MS, Kerkar N, Sirlin CB. SAFETY study: alanine aminotransferase cutoff values are set too high for reliable detection of pediatric chronic liver disease. Gastroenterology. 2010;138(4):1357-U1197.

7. Welsh JA, Karpen S, Vos MB. Increasing prevalence of nonalcoholic fatty liver disease among United States adolescents, 1988-1994 to 2007-2010. J Pediatr. 2013;162(3):496-500. e491

8. Barlow SE, Expert C. Expert committee recommendations regarding the prevention, assessment, and treatment of child and adolescent overweight and obesity: summary report. Pediatrics. 2007;120(Suppl 4):S164-92.

9. Vos MB, Abrams SH, Barlow SE, Caprio S, Daniels SR, Kohli R, Mouzaki M, Sathya P, Schwimmer JB, Sundaram SS, et al. NASPGHAN clinical practice guideline for the diagnosis and treatment of nonalcoholic fatty liver disease in children: recommendations from the expert committee on NAFLD (ECON) and the north American Society of Pediatric Gastroenterology, Hepatology and nutrition (NASPGHAN). J Pediatr Gastroenterol Nutr. 2017; 64(2):319-34

10. Kang Y, Park S, Kim S, Koh H. Estimated prevalence of adolescents with nonalcoholic fatty liver disease in Korea. J Korean Med Sci. 2018;33(14):e109.

11. Park HK, Hwang JS, Moon JS, Lee JA, Kim DH, Lim JS. Healthy range of serum alanine aminotransferase and its predictive power for cardiovascular risk in children and adolescents. J Pediatr Gastroenterol Nutr. 2013;56(6): 686-91.

12. Park HS, Han JH, Choi KM, Kim SM. Relation between elevated serum alanine aminotransferase and metabolic syndrome in Korean adolescents. Am J Clin Nutr. 2005;82(5):1046-51.

13. Song P, Yu J, Wang M, Chang X, Wang J, An L. Prevalence and correlates of suspected nonalcoholic fatty liver disease in Chinese children. Int J Environ Res Public Health. 2017;14(5):465.

14. Anderson EL, Howe LD, Jones HE, Higgins JP, Lawlor DA, Fraser A. The prevalence of non-alcoholic fatty liver disease in children and adolescents: a systematic review and meta-analysis. PLoS One. 2015;10(10):e0140908.

15. Wong WW, Chan WK, Chitturi S, Chawla Y, Dan YY, Duseja A, Fan J, Goh KL, Hamaguchi M, Hashimoto E, et al. Asia-Pacific working party on nonalcoholic fatty liver disease guidelines 2017-part 1: definition, risk factors and assessment. J Gastroenterol Hepatol. 2018:33(1):70-85.

16. Molleston JP, Schwimmer JB, Yates KP, Murray KF, Cummings OW, Lavine JE, Brunt EM, Scheimann AO, Unalp-Arida A, Network NCR. Histological abnormalities in children with nonalcoholic fatty liver disease and normal or mildly elevated alanine aminotransferase levels. J Pediatr. 2014;164(4):70713. e703

17. Vajro P, Lenta S, Socha P, Dhawan A, McKiernan P, Baumann U, Durmaz O, Lacaille F, McLin V, Nobili V. Diagnosis of nonalcoholic fatty liver disease in children and adolescents: position paper of the ESPGHAN Hepatology committee. J Pediatr Gastroenterol Nutr. 2012;54(5):700-13.

18. Park BH, Yoon JM, Kim JH, Moon JH, Lee YH, Jang SM, Kim YJ. Pathologic impact of insulin resistance and sensitivity on the severity of liver histopathology in pediatric non-alcoholic Steatohepatitis. Yonsei Med J. 2017;58(4):756-62.

\section{Publisher's Note}

Springer Nature remains neutral with regard to jurisdictional claims in published maps and institutional affiliations.

Received: 9 November 2017 Accepted: 28 June 2018

Published online: 05 July 2018

\section{References}

1. Suchy FJ, Sokol RJ, Balistreri WF. Liver disease in children, 4th edn. Cambridge. New York: Cambridge University Press; 2014. 